\title{
New Measurement of the Cosmic-Ray Positron Fraction from 5 to $15 \mathrm{GeV}$
}

\author{
J.J. Beatty, ${ }^{1, *}$ A. Bhattacharyya, ${ }^{2}$ C. Bower,${ }^{2}$ S. Coutu, ${ }^{1}$ M.A. DuVernois, ${ }^{1,}$, S. McKee, ${ }^{3}$ S.A. Minnick,, , t

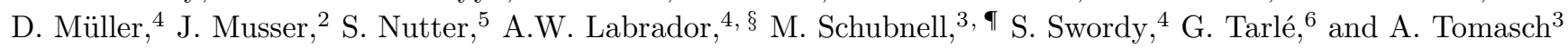 \\ ${ }^{1}$ Department of Physics, Pennsylvania State University, University Park, PA 16802 \\ ${ }^{2}$ Department of Physics, Indiana University, Bloomington, IN 47405 \\ ${ }^{3}$ Department of Physics, University of Michigan, Ann Arbor, MI 48109 \\ ${ }^{4}$ Enrico Fermi Institute and Department of Physics, University of Chicago, Chicago, IL 60637 \\ ${ }^{5}$ Department of Physics \& Geology, Northern Kentucky University, Highland Heights, KY 41099 \\ 6 Department of Physics, University of Michigan, Ann Arbor, MI 48109
}

(Dated: July 5, 2018)

\begin{abstract}
We present a new measurement of the cosmic-ray positron fraction at energies between 5 and $15 \mathrm{GeV}$ with the balloon-borne HEAT-pbar instrument in the spring of 2000. The data presented here are compatible with our previous measurements, obtained with a different instrument. The combined data from the three HEAT flights indicate a small positron flux of non-standard origin above $5 \mathrm{GeV}$. We compare the new measurement with earlier data obtained with the HEAT- $e^{ \pm}$ instrument, during the opposite epoch of the solar cycle, and conclude that our measurements do not support predictions of charge sign dependent solar modulation of the positron abundance at 5 $\mathrm{GeV}$.

PACS numbers: 11.30.Pb, 95.35.+d,96.40.Kk,98.70.Sa
\end{abstract}

Space-borne and high altitude balloon experiments have collected a considerable amount of data on the allelectron component in the cosmic radiation. Between several $\mathrm{MeV}$ and about $50 \mathrm{GeV}$ the energy spectra of electrons and positrons have been observed separately with sufficient precision to permit comparison with production and propagation models.

The relative abundances of positive and negative electrons indicate that the majority of the electron component $\left(e^{ \pm}\right)$consists of negative electrons. They are thought to be accelerated in the same Galactic sources that also generate the nuclear cosmic rays, but their observed energy spectrum is strongly affected by synchrotron and inverse Compton energy losses during propagation.

An additional component of electrons and positrons in nearly equal proportion amounts to about $10 \%$ of the total electron intensity, and is attributed to the decay of secondary particles (mostly pions) generated in hadronic interactions of cosmic-ray nuclei in the interstellar medium. These positrons constitute only a small fraction $(<0.5 \%)$ of the total observed cosmic-ray intensity, yet if they are purely secondaries they can be used as an effective probe of cosmic-ray propagation through the Galaxy. Their fraction is then expected to decline slowly with energy because of the declining path length of the primary nuclei at high rigidities.

Recent observations 1, 2, 3, 4] confirm the almost exclusively secondary nature of positrons up to a few $\mathrm{GeV}$. However, a possible structure in the positron fraction near $8 \mathrm{GeV}$ has been observed with the HEAT- $\mathrm{e}^{ \pm}$instrument [2, 5] which may defy a simple explanation.

It has been suggested that a small positron component could originate from particle interactions in nearby astrophysical sources [6, 7, 8, 9] or may be generated through the annihilation of dark matter particles in the Galactic halo 10, 11, 12, 13, 14, 15, 16, 17, 18, 19]. Such a primary positron component could lead to observable features such as those indicated by the HEAT- $\mathrm{e}^{ \pm}$measurements.

A new version of the HEAT instrument, HEAT-pbar was designed to observe the high-energy cosmic-ray antiproton flux but it is also suited for the observation of electrons and positrons at energies below $\approx 15 \mathrm{GeV}$. The instrument utilizes a multiple $\mathrm{dE} / \mathrm{dx}$ vs. rigidity technique to identify cosmic-ray particles by mass and charge. The ionization loss of relativistic particles is sampled in two stacks, each of 70 multiwire proportional chambers, filled with a $\mathrm{Xe} / \mathrm{CH}_{4}$ mixture. They are located above and below a central superconducting magnet spectrometer which measures the particle's rigidity and charge sign [20]. Scintillators at the top and bottom of the detector system measure the time of flight and, together with a scintillator just above the spectrometer, form the trigger. Unambiguous discrimination between protons, antiprotons, positrons, electrons, $\pi^{+}$and $\mu^{+}, \pi^{-}$and $\mu^{-}$, is achieved [21]. This instrument was launched from Ft. Sumner, NM on June 3, 2000 and was at float altitude for $22 \mathrm{~h}$, at an average atmospheric overburden of $7.2 \mathrm{~g} / \mathrm{cm}^{2}$. The vertical geomagnetic rigidity cut-off along the flight path varied little and averaged $4.2 \mathrm{GV}$.

The time of flight (ToF) system measures the particle velocity $\beta=v / c$ with a resolution of $\sigma_{\beta}=0.09$, permitting complete rejection of upward-going particles. The scintillator signals measure the magnitude of the particle's electric charge and select singly charged particles, with a resolution of $\sigma_{Z}=0.14$ (in charge units) for each counter.

The particle's sign of charge and rigidity $\mathrm{R}=\mathrm{pc} / \mathrm{Ze}$ are determined with the magnet spectrometer which has a 
field of about one Tesla. We retain only events with at least 13 usable tracking points in the magnet's bending plane (out of a total of 17) and at least 6 points in the non-bending plane (out of a total of 8 ).

Only the smaller $50 \%$ of the ionization signals in the 140 chambers of the multiple $\mathrm{dE} / \mathrm{dx}$ system are retained to form the restricted average signal $\left\langle d E / d x_{r e s}\right\rangle$. Owing to higher electromagnetic energy losses, $\mathrm{e}^{ \pm}$induced events tend to result in a greater number of hits in the ionization chambers of the $\mathrm{dE} / \mathrm{dx}$ system than heavier singly charged particles. Therefore we only select events with a large total number (64 or more) of channels used in calculating $\left\langle d E / d x_{r e s}\right\rangle$ and we require a minimum number of hits in the entire $\mathrm{dE} / \mathrm{dx}$ stack (155).

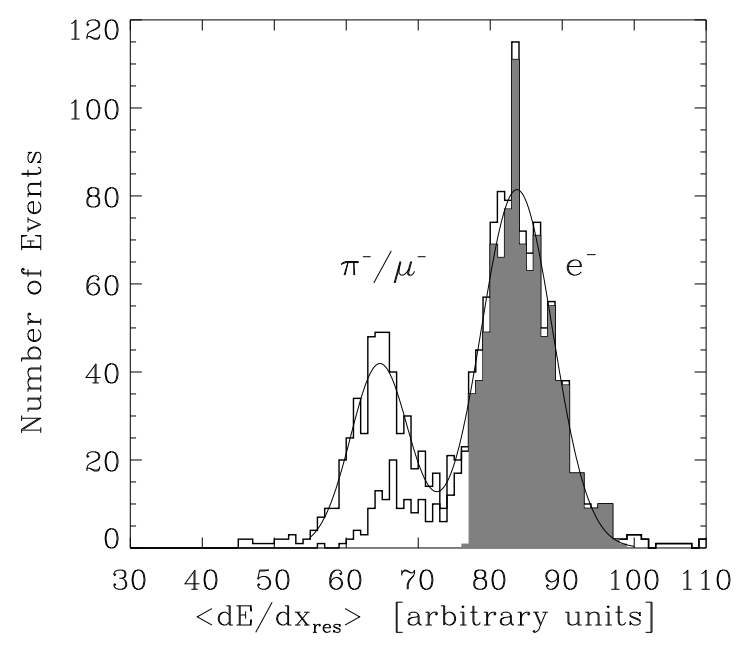

FIG. 1: Distribution of the average restricted energy loss for particles in the rigidity range $4.5-6.0 \mathrm{GV}$ identified as negatively charged. The Gaussian functions were fitted prior to the final event selection in order to obtain a well-defined association between average restricted energy loss and particle species. After the final event selection is applied, non-electron events are significantly suppressed (lower histogram). All particles within the shaded area of the histogram, determined as described in the text, are selected as electrons (and as positrons in the corresponding positive rigidity bin).

Distributions of $\left\langle d E / d x_{r e s}\right\rangle$ for particle tracks having satisfied all of the criteria are produced in three rigidity bands $(4.5-6.0,6.0-8.9$, and $8.9-14.8 \mathrm{GV})$. To illustrate the particle separation power of the instrument, the $\left\langle d E / d x_{r e s}\right\rangle$ distribution for negative particles with rigidities between $4.5 \mathrm{GV}$ and $6.0 \mathrm{GV}$ is shown in Fig. 11 Electrons are clearly separated from muons and pions whose small mass difference can not be distinguished by our instrument. Each of the three negative $\left\langle d E / d x_{\text {res }}\right\rangle$ distributions is fitted with a sum of Gaussian functions and the mean and standard deviation for the $\pi^{-} / \mu^{-}$mass peak $\left(\mathrm{m}_{\pi \mu}, \sigma_{\pi \mu}\right)$ and the electron mass peak $\left(\mathrm{m}_{e}, \sigma_{e}\right)$ are determined in each of the three rigidity bins. We define a particle as an electron (or positron) if its restricted average $\mathrm{dE} / \mathrm{dx}$ signal falls between $\left(\mathrm{m}_{\pi \mu}+3 \sigma_{\pi \mu}\right)$ and $\left(\mathrm{m}_{e}+3 \sigma_{e}\right)$ in the respective rigidity bin. To further improve the discrimination against protons and mesons we group the 140 proportional chambers into ten modules and require that the average measured energy losses in each module exceed a given threshold. This selection strongly suppresses protons and mesons while it retains almost all electrons (see Fig. (1). The residual contamination due to proton/muon/pion spillover was obtained by fitting and extrapolating the $\mathrm{p} / \mu / \pi$ distributions in Fig. 2 The background thus estimated for the three rigidity bins are $0.4,0.1,1.0$ events, respectively. Note that the selection criteria employed here were tuned to optimize the identification and statistical significance of the $\mathrm{e}^{ \pm}$peaks, and therefore differ from those employed in the antiproton analysis of the same data set 21].

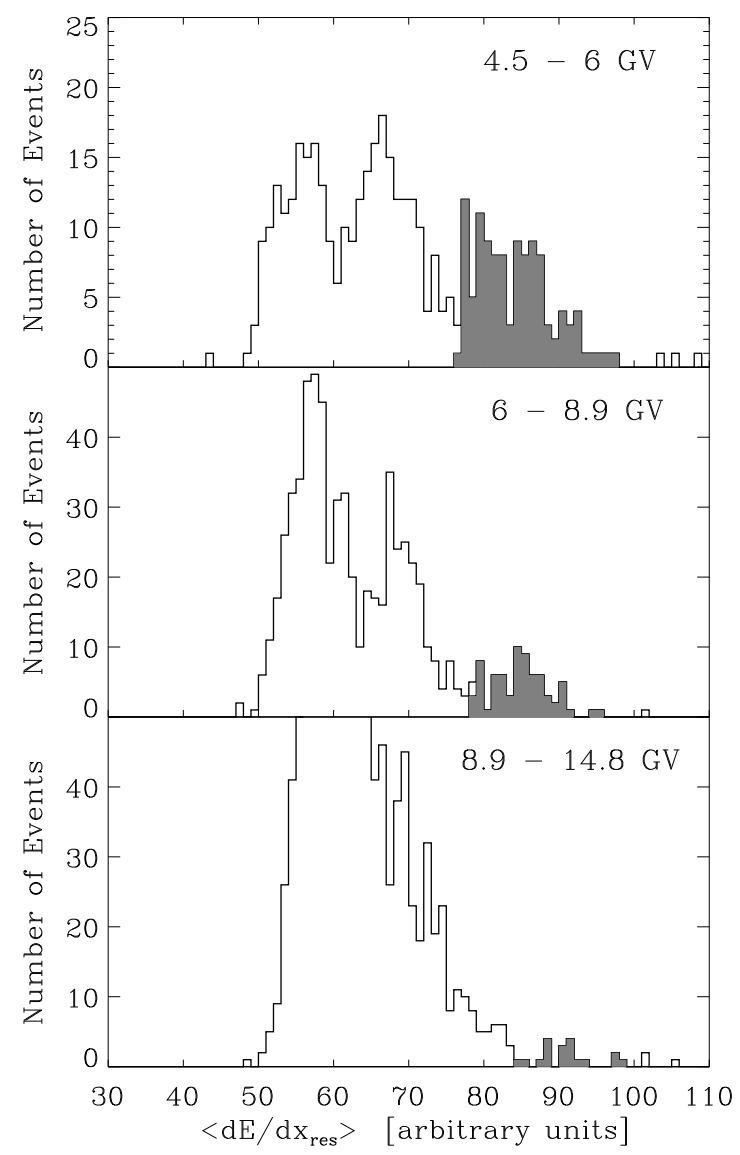

FIG. 2: Distributions of average restricted energy loss for events after all selection criteria have been applied. The three rigidity bands, $4.5-6.0 \mathrm{GV}, 6.0-8.9 \mathrm{GV}$, and $8.9-14.8 \mathrm{GV}$, are shown from top to bottom, respectively. We accept events under the shaded area as positrons. The strong relativistic rise in the energy loss for hadrons and mesons compared to electrons (which are already heavily relativistic at $\mathrm{GeV}$ energies) results in the mass peak of the particle species moving closer together with increasing rigidity and ultimately limiting the particle identification.

The atmospheric overburden during the HEAT-pbar flight varied between 4.5 and $11 \mathrm{~g} / \mathrm{cm}^{2}$, for an average 
overburden of $7.2 \mathrm{~g} / \mathrm{cm}^{2}$, which resulted in significant numbers of atmospheric secondaries. A Monte Carlo simulation, described elsewhere 22], is used to obtain the correction factors for atmospheric positron and electron production. The corrections for the positrons vary between $44 \%$ and $52 \%$, depending on energy, and those for the electrons between $5 \%$ and $6.3 \%$. Uncertainties in these atmospheric corrections result in a systematic uncertainty of about \pm 0.01 on the positron fraction.

The raw $\mathrm{e}^{ \pm}$particle counts are obtained by tallying the events with a restricted average $\mathrm{dE} / \mathrm{dx}$ signal that falls within a selected region as described above and indicated in Fig. 22 These counts are summarized in Table 【 $\left(\mathrm{N}_{e^{+}}\right.$and $\left.\mathrm{N}_{e^{-}}.\right)$They are corrected for the atmospheric secondaries, and the positron fractions calculated.

Finally, a correction factor of 1.109 is applied to rigidities at the instrument, to account on average for radiative energy losses by the electrons and positrons, and to correct to the top of the atmosphere. The correction is done in an average way, rather than on an event-by-event basis, because the events are grouped in narrow rigidity bins at the instrument prior to their identification as electrons or positrons based on their energy losses. This factor is calculated 22] as $\alpha^{t / \alpha \ln 2}$, where $t$ is the average atmospheric depth in radiation lengths and $\alpha=3.1$ is taken for the spectral index of the primary electron flux.

Fig. 3 shows the final corrected positron fractions, $e^{+} /\left(e^{+}+e^{-}\right)$, as a function of energy, compared with the previous combined HEAT- $\mathrm{e}^{ \pm}$results 2] from the 1994 and 1995 flights. The accessible energy range for our measurements is limited towards the lower energies by the geomagnetic cut-off and at high energies by the particle separation capability of the instrument. The result presented here is in agreement with the HEAT- $\mathrm{e}^{ \pm}$measurements obtained with a very different technique. The result is also compatible with the predicted ratio from 23. which assumes that all positrons are of secondary origin (dashed curve in Fig. 3). Although the new data set weakens the case for a feature at $\approx 8 \mathrm{GeV}$, an excess of positrons in this energy range cannot be ruled out. The combined dataset of the HEAT- $e^{ \pm}$and HEAT-pbar experiments is shown in Fig. 4 along with a compilation of recent measurements and predicted flux ratios.

TABLE I: Summary of event selection results and the calculated positron fraction (in $10^{-2}$ ). $\mathrm{E}_{T o A}$ is the particle kinetic energy at the top of the atmosphere. $\mathrm{N}_{e^{+}}$and $\mathrm{N}_{e^{-}}$are the number of observed positrons and electrons for each energy bin, respectively. $\mathrm{N}_{e^{+}}^{\text {cor }}$ and $\mathrm{N}_{e^{-}}^{\text {cor }}$ are the extrapolated number of positrons and electrons at the top of the atmosphere.

\begin{tabular}{lrrrrc}
\hline \hline $\mathrm{E}_{T o A}[\mathrm{GeV}]$ & $\mathrm{N}_{e^{+}}$ & $\mathrm{N}_{e^{-}}$ & $\mathrm{N}_{e^{+}}^{\text {cor }}$ & $\mathrm{N}_{e^{-}}^{\text {cor }}$ & $e^{+} /\left(e^{+}+e^{-}\right)\left[\times 10^{2}\right]$ \\
\hline $5.0-6.7$ & 112 & 902 & 55 & 845 & $6.2 \pm 0.6$ \\
$6.7-9.9$ & 71 & 712 & 40 & 673 & $5.5 \pm 0.7$ \\
$9.9-16.4$ & 18 & 238 & 8 & 226 & $3.6 \pm 0.9$ \\
\hline \hline
\end{tabular}

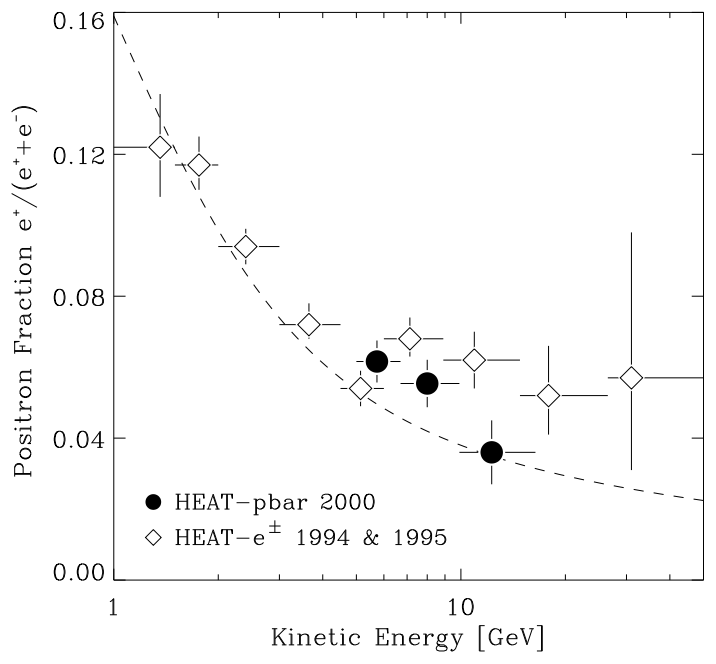

FIG. 3: The positron fraction measured by HEAT-pbar compared to measurements with a different instrument $\left(\right.$ HEAT- $\mathrm{e}^{ \pm}$ 2]); The curve shows the expectation from the model calculation in [23].

We compare our data to a computation of the cosmicray secondary positron (and electron) spectrum in a diffusive model for Galactic cosmic-ray propagation provided by 23] for different source injection spectra. With injection spectra based on the locally measured nuclear cosmic-ray spectra, the data follow the general trend of the model prediction up to a few $\mathrm{GeV}$, beyond which the observed positron fraction is higher than the calculated one. This enhancement is plausible in a variety of different theoretical scenarios.

Motivated by a measured diffuse gamma-ray spectrum above $1 \mathrm{GeV}$ that is much harder than expected [24], local cosmic-ray fluxes have been calculated based on hard proton injection spectra 23]. Interestingly, this does not only lead to a better fit of the diffuse gamma-ray data but also to an enhancement in the positron fraction above a few GeV. However, measurements of cosmic-ray antiprotons provide rather substantial evidence against the idea of explaining the diffuse gamma-ray excess with a hard nucleon spectrum 21. Therefore, if future measurements confirm the positron excess, then there may be additional sources for the cosmic-ray positrons.

An interesting suggestion is that annihilating dark matter particles in the Galactic halo, perhaps supersymmetric particles, are a source of high-energy positrons 10 , 11, 12, 13, 14, 15, 16, 17, 18, 19], and references therein). In conventional models, the lightest supersymmetric particle (e.g., neutralino) is assumed to have a mass larger than the $\mathrm{W}$ mass and direct decay will produce a substantial positron signal at about half the neutralino mass. Subsequent heavy lepton and b decays could result in an enhancement of the positron fraction at much lower energies, as shown in Fig. 44 15.

Other possible contributions to the cosmic-ray positron 


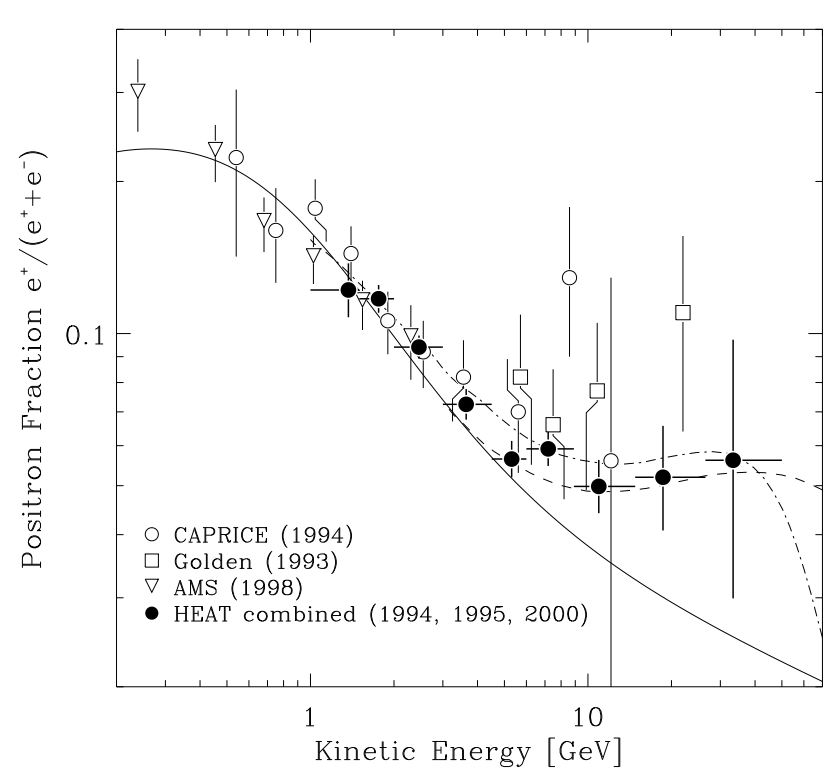

FIG. 4: The positron fraction as a function of energy for the combined HEAT- $e^{ \pm}$and HEAT-pbar data, compared to model predictions and other recent measurements ( CAPRICE 3], Golden [29], AMS [4]). Dates in parentheses give the year of the measurement and not the publication. The solid curve is the positron fraction based on a purely secondary production of positrons given by [23]. The dashed and dot dashed curves are the ratios including contributions from Higgsino LSP decay [15] and gamma-ray pulsars [6], respectively.

flux have been proposed, including synchrotron produced $\mathrm{e}^{ \pm}$pairs from Galactic pulsars [6]. Whether the apparent excess in the HEAT data is caused by one of those processes is not clear and can only be determined if further positron measurements provide improved statistical accuracy, and extend to higher energies.

It has been suggested that at energies below a few $\mathrm{GeV}$ the measured positron fraction may reflect charge sign dependent effects of the solar modulation 25, 26] and that discrepancies between results from different experiments can be related to this modulation effect. The most recent solar polar field reversal occurred just prior to the HEAT-pbar measurement and thus data from the three HEAT flights can be used to investigate this proposed effect. In the energy range $5-6 \mathrm{GeV}$ - the lowest energy common to all observations - the measured positron fraction is consistent with what we measure in 1994 and 1995 with HEAT- $e^{ \pm}$(see Fig. 3). At $5 \mathrm{GeV}$, Clem et al. 25. predict a decrease in the positron fraction by about $40 \%$, which is inconsistent with our measurements. It would be of interest to reconcile our result with the measured dependence of the antiproton flux on solar activity by the BESS group [27, 28].

In conclusion, we find that a primary contribution to the positron intensity above a few $\mathrm{GeV}$ can still not be ruled out.
This work was supported by NASA grants No. NAG 5-5058, No. NAG 5-5220, No. NAG 5-5223, and No. NAG 5-5230. We wish to thank the National Scientific Balloon Facility and the NSBF launch crews for their excellent support of balloon missions and we gratefully acknowledge contributions from D. Kouba, M. Gebhard, S. Ahmed, and P. Allison. We also thank S. Beach for essential support of the mission.

* Now at Department of Physics, Ohio State University, Columbus, OH 43210

$\dagger$ Now at School of Physics and Astronomy, University of Minnesota, Minneapolis, MN 55455

¥ Now at Department of Physics, Kent State University Tuscarawas Campus, New Philadelphia, OH 44663

$\S$ Now at California Institute of Technology, Space Radiation Laboratory, Pasadena, CA 91125

ฯ Corresponding author. Email address: schubnel@umich.edu

[1] S. Barwick et al., Phys. Rev. Lett. 75, 390 (1995).

[2] S. Barwick et al., Astrophys. J. Lett. 482, 191 (1997).

[3] M. Boezio et al., Astrophys. J 532, 653 (2000).

[4] M. Aguilar et al., Phys. Rep. 366, 331 (2002).

[5] S. Coutu et al., Astropart. Phys. 11, 429 (1999).

[6] L. Zhang and K. Cheng, Astron. Astrophys. 368, 1063 (2001).

[7] X. Chi, K. Cheng, and E. Young, Astrophys. J. Lett. 459, 83 (1996).

[8] A. Harding and R. Ramaty, Proc. 23rd ICRC 2, 92 (1987).

[9] V. Dogiel and G. Sharov, Astron. Astrophys. 229, 259 (1990).

[10] A. Tylka, Phys. Rev. Lett. 63, 840 (1989).

[11] M. Turner and F. Wilczek, Phys. Rev. D 42, 1001 (1990).

[12] M. Kamionkowski and M. Turner, Phys. Rev. D. 43, 1774 (1991).

[13] G. Jungman and M. Kamionkowski, Phys. Rep. 267, 195 (1996).

[14] G. Kane, L. Wang, and J. Wells, Phys. Rev. D 65, 057701 (2002).

[15] G. Kane, L. Wang, and T. Wang, Phys. Lett. B 536, 263 (2002).

[16] E. Baltz, J. Edsjö, K. Freese, and P. Gondolo, Phys. Rev. D 65, 063511 (2002).

[17] E. Baltz and L. Bergström, Phys. Rev. D 67, 043516 (2003).

[18] D. Hooper, J. Taylor, and J. Silk, Phys. Rev. D 69, 103509 (2004).

[19] D. Eichler and I. Maor, Astropart. Phys. 21, 195 (2004).

[20] S. Barwick et al., Nucl. Instrum. Methods A400, 34 (1997).

[21] S. Beach et al., Phys. Rev. Lett. 87, 271101 (2001).

[22] S. Barwick et al., Astrophys. J. 498, 779 (1998).

[23] I. Moskalenko and A. Strong, Astrophys. J. 493, 694 (1998).

[24] S. Hunter et al., Astrophys. J. 481, 205 (1997).

[25] J. Clem et al., Astrophys. J. 464, 507 (1996).

[26] J. Clem and P. Evenson, Astrophys. J. 568, 216 (2002).

[27] T. Maeno et al., Astropart. Phys. 16, 121 (2001). 
[28] Y. Asaoka et al., Phys. Rev. Lett. 88, 051101 (2002).

[29] R. Golden et al., Astrophys. J. Lett. 457, 103 (1996). 